\title{
Discrete Element Method Simulation of a Boulder Extraction from an Asteroid
}

\author{
Anton V. Kulchitsky ${ }^{1}$, Jerome B. Johnson ${ }^{1}$, David Reeves ${ }^{2}$ and Allen Wilkinson ${ }^{3}$ \\ ${ }^{1}$ University of Alaska Fairbanks, Institute of Northern Engineering, P.O.Box 755910, \\ Fairbanks, AK 99775-5910, Email: anton.kulchitsky@alaska.edu \\ ${ }^{2}$ NASA Langley Research Center, Space Mission Analysis Branch, 1 N. Dryden St. \\ Mail Stop 462, Hampton, VA 23681, Email: david.m.reeves@nasa.gov \\ ${ }^{3}$ Chaff LLC, 1286 Yellowstone Rd., Cleveland Heights, OH 44121, \\ Email: aw@chaff.biz
}

\begin{abstract}
The force required to pull $7 \mathrm{t}$ and $40 \mathrm{t}$ polyhedral boulders from the surface of an asteroid is simulated using the discrete element method considering the effects of microgravity, regolith cohesion and boulder acceleration. The connection between particle surface energy and regolith cohesion is estimated by simulating a cohesion sample tearing test. An optimal constant acceleration is found where the peak net force from inertia and cohesion is a minimum. Peak pulling forces can be further reduced by using linear and quadratic acceleration functions with up to a $40 \%$ reduction in force for quadratic acceleration.
\end{abstract}

\section{Introduction}

Based on the concept of redirecting a Near Earth Asteroid (NEA) to the Earth-Moon vicinity (Brophy, Culick, Friedman, Allen, Baughman, \& Bellerose, 2012), NASA is developing the Asteroid Redirect Mission (ARM) to identify, capture and redirect an asteroid to a safe lunar orbit for future exploration. Two concepts are proposed. The first concept is to capture and redirect an entire small asteroid and bring it to lunar orbit for future study by astronauts and robotic missions. The second concept is to retrieve a boulder from a larger asteroid and return it to the same lunar orbit.

The boulder retrieval operation contains many uncertainties. One of the problems that arises is the need to identify the forces required to separate the boulder from the surface of the asteroid. The magnitude of these extraction forces can be affected by the size and shape of the boulder, the strength of the regolith and the depth to which it covers the boulder, the acceleration imparted to the boulder during extraction, and the mass forces of gravitational attraction and apparent centrifugal force due to asteroid rotation. Regolith strength is affected by its packing density, grain size and shape distribution, grain contact friction, degree of grain interlocking, gravitation attraction of each particle with the mean field gravitational force of the rest of the asteroid, electrostatic forces, solar radiation pressure, and van der Waals cohesive forces between regolith grains and between the grains and boulder. The strength can also be affected by cold welding and the presence of water or ice in the asteroid as recent observations of water vapors around Ceres (Küppers, 2014) may indicate. Van der Waals cohesive forces are considered to be a dominant contributor to regolith strength, competing with regolith weight, but greater than electrostatic and solar radiation pressure forces (Scheeres, Hartzell, Sanchez, \& Swift, 2010). 
The goal of this work is to use the COUPi (Controlled Objects Unbound Particles interaction) discrete element method (DEM) model (Kulchitsky, Nye, \& Johnson, 2013; Nye, Kulchitsky, \& Johnson, 2014) to perform simulations of boulder retrieval in the asteroid surface reference frame and calculate the reaction forces.

\section{Contact Model Description}

In the COUPi DEM model, the contact forces are represented by normal elastic forces, adhesive forces, tangential forces and normal and tangential damping forces. The forces are computed using a modified Hertz-Mindlin model. The detailed explanation of COUPi DEM contact physics is described in (Nye, Kulchitsky, \& Johnson, 2014). The normal force component depends on the overlap between particles $(\delta)$. The elastic component of the contact force can be found as follows:

$$
F_{n e}=\frac{4}{3} E_{*} r_{*}^{1 / 2} \delta^{3 / 2},
$$

where the effective Young's modulus $E_{*}$ and effective interaction radius $r_{*}$ are defined as follows:

$$
r_{*}=\left(1 / r_{1}+1 / r_{2}\right)^{-1}, \quad E_{*}=\left(\left(1-v_{1}^{2}\right) / E_{1}+\left(1-v_{2}^{2}\right) / E_{2}\right)^{-1}
$$

where $r_{1}$ and $r_{2}$ are the radii of contacting particles curvature at the contact point, $v_{1}$ and $v_{2}$ are Possion's ratios, and $E_{1}$ and $E_{2}$ are Young's moduli of contacting materials. The normal force damping component is used from the extended Hertz theory (Kuwabara \& Kono, 1987):

$$
F_{n i}=k_{n i} \delta^{1 / 2} d \delta / d t
$$

where the constant damping coefficient $k_{n i}$ nonlinearly depends on the coefficient of restitution (Müller \& Pöschel, 2011).

The adhesive normal component of the contact force is calculated using Johnson, Kendall, and Roberts (JKR) model (Johnson, Kendall, \& Roberts, 1971; Johnson, 2003) with linearization proposed by (Brilliantov \& Pöschel, 2004; Brilliantov, Albers, Spahn, \& Pöschel, 2007):

$$
F_{n c}=(4 \sqrt{6 \pi} / 9) \gamma^{1 / 2} E_{*}^{1 / 2} r_{*}^{3 / 4} \delta^{3 / 4},
$$

where $\gamma$ is surface energy value for interacting materials.

The tangential force value $\boldsymbol{F}_{\tau}^{t+\Delta t}$ at each time step $t+\Delta t$ is calculated incrementally from the force at the previous time step $t$ similar to (Zhao, Nezami, Hashash, \& Ghaboussi, 2006; Nye, Kulchitsky, \& Johnson, 2014) using the following expression:

$$
\begin{gathered}
\boldsymbol{F}_{\tau}^{t+\Delta t}=\xi \boldsymbol{\Phi}_{\tau}^{t+\Delta t}, \quad \xi=\min \left(1, \mu F_{n} /\left|\boldsymbol{\Phi}_{\tau}^{t+\Delta t}\right|\right) . \\
\boldsymbol{\Phi}_{\tau}^{t+\Delta t}=\boldsymbol{F}_{\tau}^{t}-\left(\boldsymbol{F}_{\tau}^{t} \cdot \boldsymbol{n}^{t}\right) \boldsymbol{n}^{t}-k_{\tau} \boldsymbol{v}_{\tau}^{t+\Delta t} \Delta t,
\end{gathered}
$$

where $\mu$ is the Coloumb coefficient of friction between two particles, $k_{\tau}$ is the tangential stiffness, $\boldsymbol{n}^{t}$ is the normal vector to the contact plane at time $t$, and $\boldsymbol{v}_{\tau}^{t+\Delta t}$ is the relative velocity of the particle at the point of contact. 


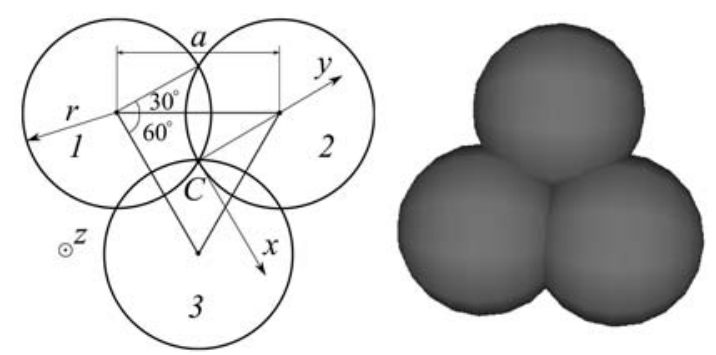

Figure 1. Tri-spherical particles used in simulations.

\section{Particle Shape}

To simulate the regolith, we used a monodisperse system of particles created with 3 equal spheres symmetrically connected together such that they have only one common point as shown in Figure 1.

\section{Cohesion Scaling}

\section{Introduction}

The cohesive force at the contact in the JKR model is defined by the surface energy parameter $\gamma$ and the effective curvature radius $r_{*}$ of the particles at the point of contact and depends on the type of the contact and other material and contact properties. It can be shown (Johnson, 2003) that the maximum attracting force at the contact in JKR model is proportional to both $\gamma$ and $r_{*}$ and does not depend on other material properties:

$$
F_{\text {max }} \sim \gamma r_{*}
$$

Any pulling force stronger than this will result in the failure of a static contact. Thus, $F_{\max }$ is the strength of the cohesive force between the particles. Strength $F_{\max }$ is a local parameter that determines the strength of a single contact. This parameter needs to be linked to the macroscopic regolith cohesive strength defined as the maximum pulling stress a regolith sample can have at any point (Li, Zeng, \& Wilkinson, 2013). The stress in granular material is defined on a surface within the granular material that intersects a large enough number of particles.

DEM simulations are performed with particles that are significantly larger than actual regolith particles due to computational limitations. As particles become larger the cohesive forces become smaller according to Eq. ( 1 ). Thus the surface energy $\gamma$ needs to be scaled up to accommodate this difference in size. For small values of surface energy, the standard triaxial compression test might not be sensitive enough (Modenese, Utili, \& Houlsby, 2012). To link the cohesion stress with $\gamma$ and $r_{*}$ we designed a cohesion gravity test as described below. 

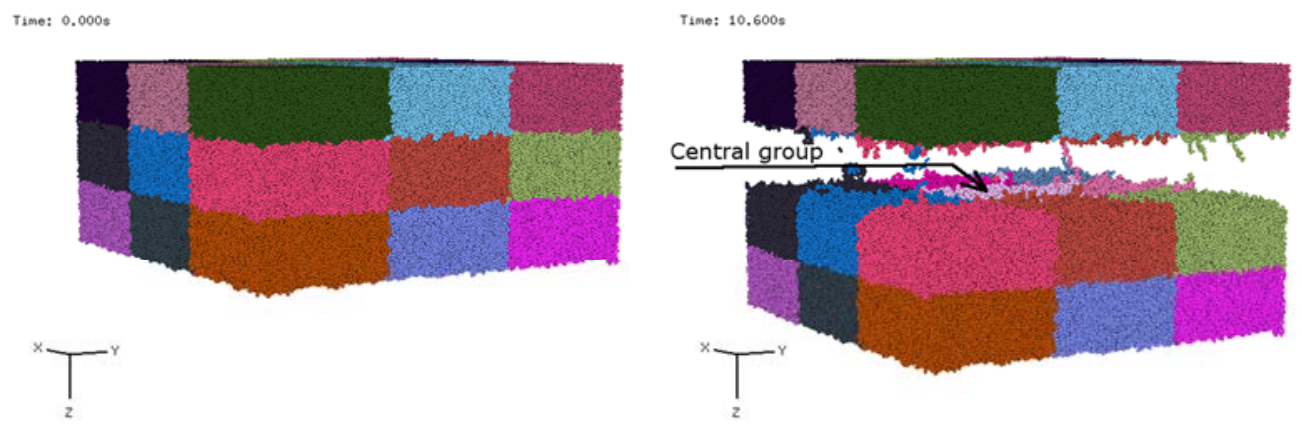

Figure 2. Cohesion gravity test. Left panel: initial setup, Right panel: final stage.

Cohesion test description

The main goal for this test is to measure the separation cohesion stress in granular materials depending on the surface energy and particle size. The test uses an increasing gravitational field to ramp up a uniaxial internal tensile stress in the regolith to induce failure. We keep the packing density the same for all the tests. The cohesion test consists of the following stages:

1. Filling the particles. The goal for this stage is to fill a box with 27 groups of particles such that each group forms a connected rectangular specimen as shown in Figure 2 (left panel, each group shown with its own color, the specimen is shown upside down). To do this, 3 layers of identical tri-spherical particles with 9 groups in each layer are filled one by one. To fill a layer, the particles are put into the grid above the previous layer or the bottom of the box so they do not overlap. Then, they fall under the gravity $10 \mathrm{~m} / \mathrm{s}^{2}$. At this stage there is no cohesion set between the particles, e.g. $\gamma=0$. The Coulomb friction is chosen to control the packing ratio for the particles.

2. Cohesion application. The goal of this stage is to equilibrate the particles with surface energy set to the desired values. The surface energy is a property of the materials interacting and can be set up for each pair of materials in the system. We use the following rules for setting the surface energy:

a. The two upper layers consist of particles with the desired surface energy $\gamma$. There is neither friction nor adhesion set between those layers and the box walls to avoid their interaction.

b. The 9 groups of the lowest layer (shown on top in Figure 2) have 10 times higher surface energy between its particles. It has normal surface energy $\gamma$ between its particles and the particles from other layers. It has 10 times higher cohesion between the layer and the wall of the box. The meaning of this set up is explained below.

The material equilibrates for a few seconds to adjust to the new surface energy values.

3. Gravity relaxation. The goal for this stage is to remove the gravitational forces. Due to the potential energy stored in each contact, the gravity cannot be removed immediately. Instead, a linear gravity relaxation from initial value of $10 \mathrm{~m} / \mathrm{s}^{2}$ to 0 within $10 \mathrm{~s}$ is applied. After this the specimen is left for $20 \mathrm{~s}$ to equilibrate in 0 gravity conditions. 


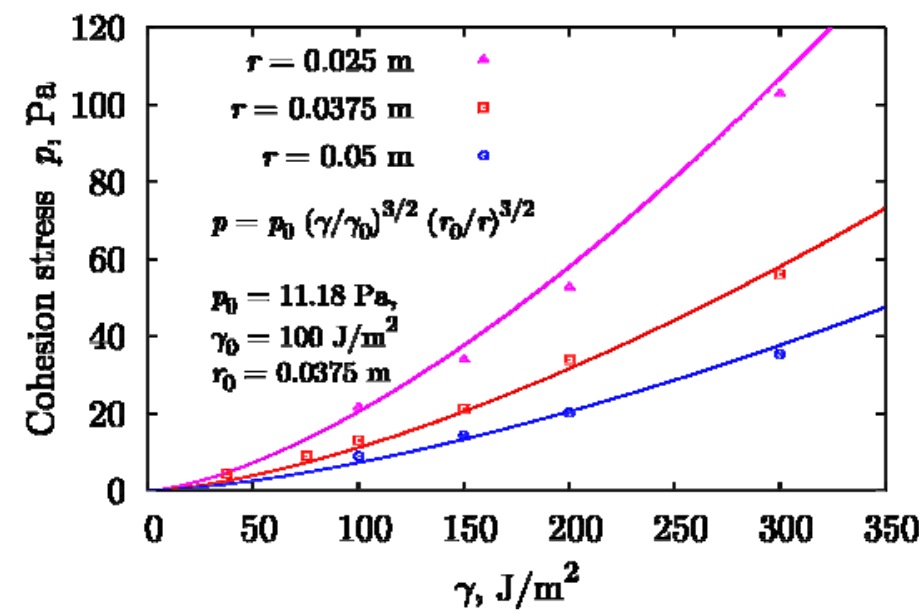

Figure 3. Cohesion stress as a function of surface energy for different radii.

4. Gravity pulling. At this stage a negative gravity that is linearly increasing with time is applied until the sample breaks as shown in Figure 2 (right panel). As particles on the lowest level have significantly stronger bonds, the breakage occurs between layer 1 and layer 2 where the maximum hydrostatic pulling stress occurs. Moreover, the particles that are strongly connected to the box do not separate from the box. The net force vector between central groups of particles between layer 1 and layer 2 is recorded as a function of time. The pulling stress between those groups is calculated by dividing the net force acting from the particles of one group acting on the particles of the other group by the cross-sectional surface area separating those groups. The maximum pulling stress is recorded as a cohesion stress for this specimen. The groups are chosen in the center to avoid any possible boundary effects as shown in Figure 2.

\section{Cohesion test results}

We tested the particles from $0.025 \mathrm{~m}$ to $0.05 \mathrm{~m}$ to find how cohesion stress depends on the surface energy and the radius. The results are shown in Figure 3. We found that for the range of cohesion stress we are interested in and for a packing density between 0.57 and 0.58 , a good approximation for cohesion stress can be given by the following formula:

$$
p=p_{0}\left(\gamma / \gamma_{0}\right)^{3 / 2}\left(r_{0} / r\right)^{3 / 2},
$$

where $p_{0}, \gamma_{0}, r_{0}$ are some constants as shown in Figure 3.

\section{Simulation Setup}

The simulation consists of the following stages.

1. Filling the particles. The goal for this stage is to fill a box with particles. The particles are put into the grid above the bottom of the box so they do not overlap. They are then allowed to fall due to gravity at $10 \mathrm{~m} / \mathrm{s}^{2}$. At this stage there is no cohesion set between the particles, e.g. $\gamma=0$. The Coulomb friction is chosen to control the packing ratio for the particles as in the 
Table 1. Material and contact properties and simulation parameters used in the tests. The material stiffness was reduced to increase the time step for the computations.

\begin{tabular}{l|l}
\hline Material/Contact/Simulation Property & Value \\
\hline Material density, $\rho, \mathrm{kg} / \mathrm{m}^{3}$ & 2875.0 \\
Packing density & 0.57 (approximately) \\
Stiffness, $G, \mathrm{GPa}$ & 0.1 \\
Poisson coefficient, $v$ & 0.25 \\
Interparticle friction ratio, $\mu$ & 0.3 \\
Coefficient of restitution $($ at $1 \mathrm{~m} / \mathrm{s}), C_{R}$ & 0.1 \\
Tangential contact stiffness, $k_{\tau}$ & 0.7 \\
Boulder mass & $7 \mathrm{t}$ and $40 \mathrm{t}$ \\
Boulder equivalent sphere diameter & $1.67 \mathrm{~m}$ and $2.98 \mathrm{~m}$ \\
Number of particles for cohesion tests & $38 \mathrm{~K}, 81 \mathrm{~K}$, and $302 \mathrm{~K}$ \\
Number of particles for boulder $7 \mathrm{t}$ test & $27,380(82 \mathrm{~K}$ spheres) \\
Number of particles for boulder $40 \mathrm{t}$ test & $176 \mathrm{~K}(528 \mathrm{~K}$ spheres) \\
\hline
\end{tabular}

cohesion test. The polyhedral boulder of a realistic shape is planted in the upper layers and is deposited together with the particles.

2. Cohesion application. The goal of this stage is to equilibrate the particles with surface energy set to the desired values.

3. Gravity adjustment. The goal for this stage is to relax the gravity forces to the forces estimated on the asteroid. Similar to the cohesion test, the linear gravity relaxation from initial value of $10 \mathrm{~m} / \mathrm{s}^{2}$ to $2.5 \cdot 10^{-5} \mathrm{~m} / \mathrm{s}^{2}$ within $10 \mathrm{~s}$ is applied. After this the specimen is left for $20 \mathrm{~s}$ to equilibrate in new gravity conditions.

4. Pulling the boulder. The boulder is pulled with controlled vertical velocity. The forces acting on the boulder from the regolith are recorded. The vertical velocity can be an arbitrary function of time and were set to grow linearly, quadratically or cubically to a particular constant value.

The material, contact and simulation parameters are shown in Table 1.

\section{Tests}

The tests were performed for two sample boulders with mass $7 \mathrm{t}$ with equivalent sphere diameter of $1.67 \mathrm{~m}$ and $40 \mathrm{t}$ with equivalent sphere diameter of $2.98 \mathrm{~m}$ based

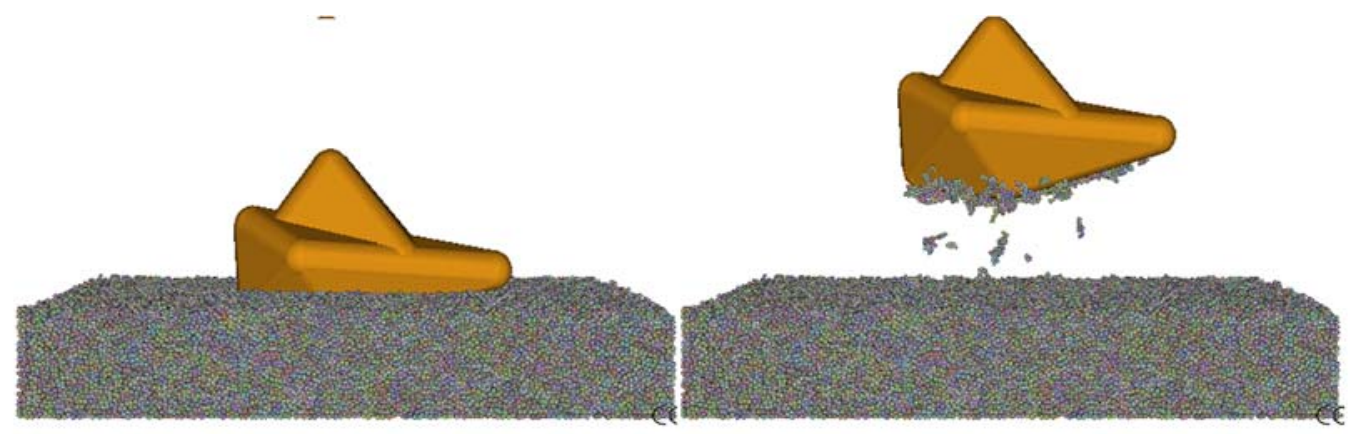

Figure 4. Pulling the boulder visualization. 
on estimations of the boulder sizes suitable for ARM mission (Mazanek \& Merrill, 2013). The goal was to establish the maximum pulling force depending on the estimated cohesion stress of the regolith as well as the time dependence of the pulling force for different methods of pulling. The boulder was planted shallow with less than $30 \%$ being under the surface. Then, vertical acceleration was applied until the boulder reached the velocity of $0.2 \mathrm{~m} / \mathrm{s}$. The force vector acting on the boulder from the particles was recorded. The absolute value of the force vector is considered to be the force applied to the boulder due to cohesion in the regolith. The test configuration is shown in Figure 4.

Table 2. Simulations of the $40 t(40,000 \mathrm{~kg})$ boulder pull.

\begin{tabular}{cccccccc}
\hline Test & $\boldsymbol{\gamma}, \mathbf{J} / \mathbf{m}^{\mathbf{2}}$ & $\boldsymbol{p}_{\boldsymbol{c}}, \mathbf{P a}$ & $\boldsymbol{t}_{\boldsymbol{f}, \mathbf{S}}$ & $\boldsymbol{a}, \mathbf{m} / \mathbf{s}^{\mathbf{2}}$ & $\boldsymbol{v}_{\mathbf{f}}, \mathbf{m} / \mathbf{s}$ & $\boldsymbol{F}_{\mathbf{c}}, \mathbf{N}$ & $\boldsymbol{F}_{\mathbf{t}}, \mathbf{N}$ \\
\hline 1 & 165 & 25 & 4 & 0.05 & 0.2 & 287.22 & 2287.22 \\
2 & 915 & 350 & 4 & 0.05 & 0.2 & 1676.00 & 3676.00 \\
\hline
\end{tabular}

Table 3. Simulations of the $7 \mathrm{t}(6974 \mathrm{~kg})$ boulder pull. Notation: $p_{c}$ is estimated cohesion stress, $t_{f}$ is total pulling time, $a$ is acceleration, $v_{\mathrm{f}}$ is final velocity of the boulder, $F_{\mathrm{c}}$ is maximum force from the cohesion, $F_{\mathrm{t}}$ is the maximum total force

\begin{tabular}{cccccccc}
\hline Test & $\boldsymbol{\gamma}, \mathbf{J} / \mathbf{m}^{\mathbf{2}}$ & $\boldsymbol{p}_{\boldsymbol{c}}, \mathbf{P a}$ & $\boldsymbol{t}_{\boldsymbol{f}, \mathbf{S}}$ & $\boldsymbol{a}, \mathbf{m} / \mathbf{s}^{\mathbf{2}}$ & $\boldsymbol{v}_{\mathbf{f}}, \mathbf{m} / \mathbf{s}$ & $\boldsymbol{F}_{\mathbf{c}}, \mathbf{N}$ & $\boldsymbol{F}_{\mathbf{t}}, \mathbf{N}$ \\
\hline 1 & 165 & 25 & 8 & 0.025 & 0.2 & 48.48 & 222.83 \\
2 & 460 & 125 & 8 & 0.025 & 0.2 & 237.45 & 411.80 \\
\hline 3 & 915 & 350 & 8 & 0.025 & 0.2 & 958.75 & $1,122.1$ \\
4 & 915 & 350 & 4 & 0.05 & 0.2 & 615.62 & 964.32 \\
5 & 915 & 350 & 2 & 0.1 & 0.2 & 691.22 & $1,388.62$ \\
6 & 915 & 350 & 8 & $0.25 t$ & 0.2 & 824.53 & 928.12 \\
7 & 915 & 350 & 8 & $6.25 \cdot 10^{-3} t^{2} / 2$ & 0.2 & 524.33 & 555.00 \\
\hline 8 & 2600 & 1650 & 8 & 0.025 & 0.2 & 2330.42 & 2504.77 \\
\hline
\end{tabular}

The primary set of tests was performed with constant vertical acceleration. A Linear and quadratic vertical acceleration pull functions were also applied for $7 \mathrm{t}$ boulder. The results of the simulations are represented in Table 2 and Table 3 . The cohesion strength was varied from $100 \mathrm{~Pa}$ to $2000 \mathrm{~Pa}$ to cover a wide range of potential extraction forces.

\section{Discussion}

The simulation runs for the $7 \mathrm{t}$ boulder with cohesion strength of $350 \mathrm{~Pa}$ show that for constant acceleration runs there is an optimal acceleration when the maximum cohesion force value $\boldsymbol{F}_{\mathbf{c}}$ reaches its minimum (test 4 ). Even though the inertial force doubles when the acceleration increases from $0.025 \mathrm{~m} / \mathrm{s} 2$ to $0.05 \mathrm{~m} / \mathrm{s} 2$, the total force is still smaller for the higher acceleration case due to the lower cohesion force.

Another finding is that application of linear or quadratic acceleration functions produce smaller maximum cohesion forces than constant acceleration. Figure 5 shows the force from cohesion acting on the $7 \mathrm{t}$ boulder from regolith during the pull process for constant, linear, and quadratic acceleration functions. The peak value of the forces is lowest in the quadratic acceleration case. The force both develops and 
drops off slower than in constant acceleration case, but results in a $40 \%$ reduction in maximum force.

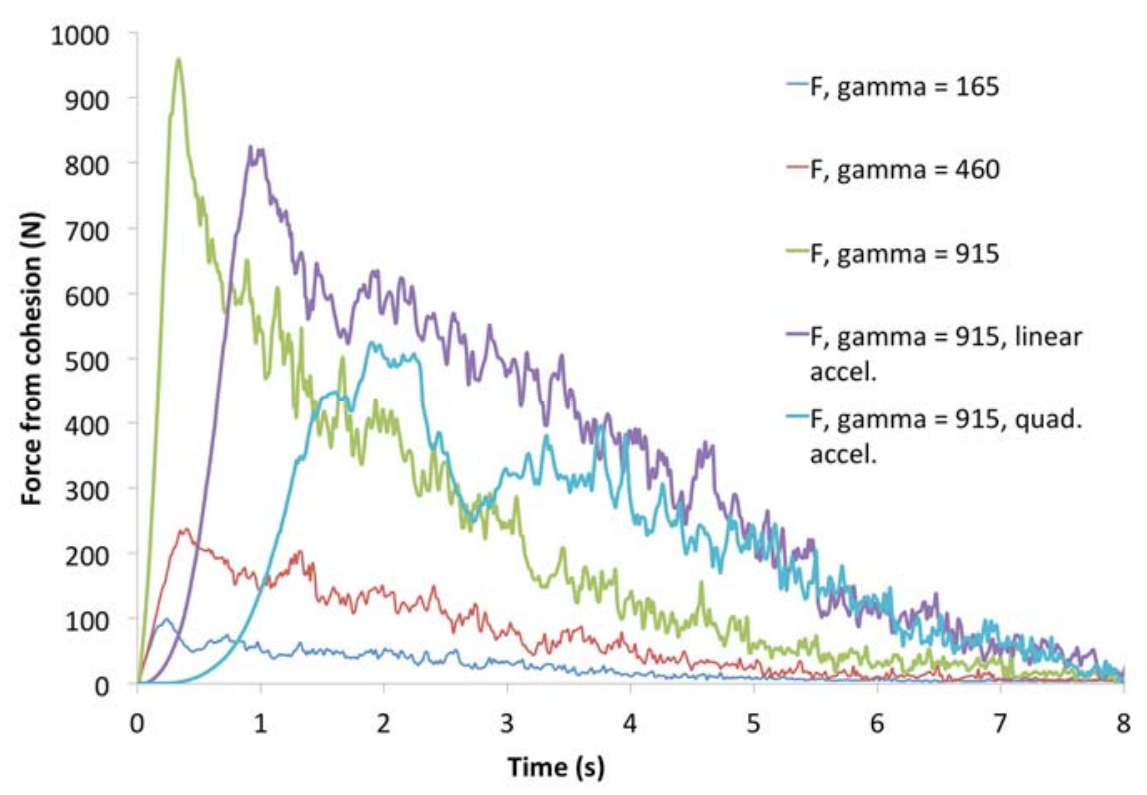

Figure 5. Force from regolith during the 8 s pull tests for $7 \mathrm{t}$ boulder.

Figure 5 also shows the forces with smaller cohesion strength (tests 1 and 2). We can expect that cohesive forces acting on a larger boulder will grow proportionally to the number of contacts and hence to the area of the boulder that is in contact with the regolith. Thus, we can estimate that the maximum force for a larger asteroid will also grow proportionally to its surface area and assume that

$$
F_{c 1} / d_{1}^{2}=F_{c 2} / d_{2}^{2}
$$

for the same pulling method. Where $F_{c 1}$ and $F_{c 2}$ are the maximum reaction forces from the regolith for the first and second boulder accordingly, and $\mathrm{d}_{1}$ and $\mathrm{d}_{2}$ are boulder characteristic diameters. Comparing test 4 from Table 3 to test 2 from Table 2 for similar pulling acceleration conditions shows very good agreement with the above formula. Thus, the forces for larger boulders can be estimated from simulations of smaller boulders, which can greatly reduce the computational effort of determining the extraction force of large boulders.

\section{Conclusions}

We considered the problem of using a discrete element method simulation to estimate the forces that appear when pulling a boulder from an asteroid in microgravity conditions where cohesion forces are considered to be a significant factor. Simulations of $7 \mathrm{t}$ and $40 \mathrm{t}$ boulder extractions from an asteroid surface depend on the pulling method, cohesion strength of the regolith and boulder size. As the maximum force from cohesion depends on both the surface energy and geometric size of the grains, and we cannot model actual grain sizes due to computational limitations, we 
have to introduce an artificial surface energy depending on the particle size to match the desired cohesion strength of the regolith. To do this, we developed a cohesion strength test that uses slowly increasing artificial gravity to tear apart a regolith sample. The cohesion strength is then measured as a maximum stress within the sample during the tearing process. Using this data, we match necessary surface energy with cohesion strength in regolith.

We developed a method to create a particle bed in the DEM model and perform a simulation of a boulder extraction in microgravity conditions. Test pulls of $7 \mathrm{t}$ and $40 \mathrm{t}$ boulders from an asteroid surface were performed for different boulder accelerations. It was found that the maximum cohesive force from the regolith depends on the method and speed of the pulling. For a constant acceleration pull there is the possibly that an optimal acceleration exists where the net maximum force from inertia and cohesion are smallest. Further reductions in pulling forces can be achieved by using linear and quadratic acceleration functions with up to a $40 \%$ reduction in force for quadratic acceleration.

Comparison of extraction forces for the $7 \mathrm{t}$ boulder with the $40 \mathrm{t}$ boulder indicated that the extraction force for a larger boulder, using the same pulling method can be estimated by multiplying the smaller boulder extraction force by the ratio of square of characteristic diameter of the larger boulder to a square of the diameter of the smaller boulder:

$$
F_{c 1} / d_{1}^{2}=F_{c 2} / d_{2}^{2}
$$

\section{Acknowledgements}

The authors would like to thank the NASA Asteroid Redirect Robotic Mission (ARRM) Alternate Concept Team and Analytical Mechanics Associates, Inc. for their funding and support during this work.

This work (publication SSERVI-2014-169) was partially supported by NASA's Solar System Exploration Research Virtual Institute.

\section{Bibliography}

Brilliantov, N. V., \& Pöschel, T. (2004). Collision of adhesive viscoelastic particles. In H. Hinrichsen, \& D. Wolf (Eds.), The physics of granular media (pp. 1-22). Weinheim, FRG: Wiley-VCH Verlag GmbH \& Co.

Brilliantov, N. V., Albers, N., Spahn, F., \& Pöschel, T. (2007). Collision dynamics of granular particles with adhesion. Phys. Rev. E., 76(5), 051302.

Brophy, J., Culick, F., Friedman, L., Allen, C., Baughman, D., \& Bellerose, J. (2012). Asteroid retrieval feasibility study. Keck Institute for Space Studies, Califonia Institute of Technology, Jet Propulsion Laboratory.

Johnson, K. L. (2003). Contact Mechanics (9 ed.). Cambridge: Cambridge University Press.

Johnson, K. L., Kendall, K., \& Roberts, A. D. (1971). Surface energy and the contact of elastic solids. Proceedings of the Royal Society of London A8, 324(1558), 301-313. 
Kulchitsky, A. V., Nye, B., \& Johnson, J. B. (2013). Intersecting dilated convex polyhedra method for representing general polyhedral particles in COUPi DEM model. In G. Mustoe (Ed.), 6th International Conference on Discrete Element Methods and related techniques (pp. 469-474). Golden: Colorado School of Mines.

Küppers, Michael, et al. "Localized sources of water vapour on the dwarf planet (1) Ceres". Nature, no. 505 (2014), 525-527.

Kuwabara, G., \& Kono, K. (1987). Restitution coefficient in a collision between two spheres. Japanese Journal of Applied Physics, 26(8), 1230-1233.

Li, Y., Zeng, X., \& Wilkinson, A. (2013, 10). Measurement of small cohesion of JSC-1A lunar simulant. Journal of Aerospace Engineering, 26(4), 882-886.

Mazanek, D., \& Merrill, G. (2013, December 17). ARRM alternate approach midterm status briefing. NASA LaRC.

Modenese, C., Utili, S., \& Houlsby, G. T. (2012). A Study of the Influence of Surface Energy on the Mechanical Properties of Lunar Soil Using DEM. In K. Zachny (Ed.), Earth and Space 2012 (pp. 45-54). ASCE.

Müller, P., \& Pöschel, T. (2011, August). Collision of viscoelastic spheres: Compact expressions for the coefficient of normal restitution. Physical Review E, 84(2), 021302.

Nye, B., Kulchitsky, A. V., \& Johnson, J. B. (2014). Intersecting dilated convex polyhedra method for modeling complex particles in discrete element method. International Journal for Numerical and Analytical Methods in Geomechanics.

Scheeres, D. J., Hartzell, C., Sanchez, P., \& Swift, M. (2010). Scaling forces to asteroid surfaces: The role of cohesion. ICARUS, 210(2), 968-984.

Zhao, D., Nezami, E. G., Hashash, Y. M., \& Ghaboussi, J. (2006). Three-dimensional discrete element simulation for granular materials. Engineering Computations, 7(23), 749-770. 